\title{
Design, Development and Testing of an Air Damper to Control the Resonant Response of a SDOF Quarter-Car Suspension System
}

\author{
Ranjit G. Todkar \\ Department of Mechanical Engineering, P.V.P. Institute of Technology, Sangli, India \\ E-mail:rgtodkar@gmail.com \\ Received October 21, 2011; revised November 3, 2011; accepted November 10, 2011
}

\begin{abstract}
An air damper possesses the advantages that there are no long term changes in the damping properties, there is no dependence on working temperature and additionally, it has less manufacturing and maintenance costs. As such, an air damper has been designed and developed based on the Maxwell type model concept in the approach of Nishihara and Asami and Nishihara [1]. The cylinder-piston and air-tank type damper characteristics such as air damping ratio and air spring rate have been studied by changing the length and diameter of the capillary pipe between the air cylinder and the air tank, operating air pressure and the air tank volume. A SDOF quarter-car vehicle suspension system using the developed air enclosed cylinder-piston and air-tank type damper has been analyzed for its motion transmissibility characteristics. Optimal values of the air damping ratio at various values of air spring rate have been determined for minimum motion transmissibility of the sprung mass. An experimental setup has been developed for SDOF quarter-car suspension system model using the developed air enclosed cylinder-piston and air-tank type damper to determine the motion transmissibility characteristics of the sprung mass. An attendant air pressure control system has been designed to vary air damping in the developed air damper. The results of the theoretical analysis have been compared with the experimental analysis.
\end{abstract}

Keywords: Ride Comfort, Quarter-Car Suspension Model, Cylinder-Piston and Air-Tank Type Air Damper, Motion Transmissibility, Optimal Air Damping Ratio

\section{Introduction}

The control of response of the sprung mass of a SDOF quarter-car suspension system subjected to the road excitation is necessary in the neighborhood of the resonance for the better ride comfort, road holding and stability. Various damping mechanisms such as, hydraulic, electromagnetic, ER and MR fluid and air dampers have been reported in the literature [1-3]. In this paper, an air enclosed cylinder-piston and air-tank type air damper configuration has been selected for design and development because in these dampers there are no long term changes in the damping properties, no dependence on working temperature. Air dampers have less manufacturing and maintenance costs. A SDOF quarter-car vehicle suspension model using such a developed air damper has been analyzed for its motion transmissibility characteristics. The air damper has been designed and developed as a
Maxwell type model in the approach of Nishihara and Asami and Nishihara [1]. The air damper characteristics such as air damping ratio and air spring rate have been studied. Optimal values of the air damping ratio at various values of air spring rate have been determined to obtain minimum motion transmissibility of the sprung mass. An experimental setup for SDOF quarter-car suspension system using the developed air enclosed cylinder-piston and air-tank type damper has been developed with an attendant air pressure control system.

\section{Development of an Air Enclosed Cylinder-Piston and Air-Tank Type Damper}

A cylinder-piston and air-tank type damper, both the sides of which are connected to two surge tanks through capillary pipes has been developed. The arrangement is used 
to set the desired damping properties by allowing the changes in 1) Tank volume to cylinder volume ratio $N t, 2$ ) Operating air pressure $p i$, and 3) Capillary pipe length $l_{\text {pipe }}$ and diameter $d_{\text {pipe }}$. Figure 1(a) shows a cylinderpiston and air-tank type air damper. Figure 1(b) shows the mathematical models for the air Damper [4].

\subsection{Air Spring Rate $\boldsymbol{k}_{a}$ [3]}

$$
k_{a}=\frac{2 n p i s^{2}}{v_{t}}=\left[\frac{2 n s^{2}}{v_{c}}\right]\left[\frac{p i}{N t}\right]
$$

where $\quad v_{t}=v_{c} N t$

where $n$ is the index of expansion of air, $p i$ is the operating air pressure in the system, $s$ is the cross sectional area of the piston, $v_{t}$ is the air tank volume and $v_{c}$ is the air cylinder volume.

Let air spring rate ratio $k=\left[\frac{k_{a}}{k_{1}}\right]$

where $k_{1}$ is the suspension spring rate.

Substituting the value of $k_{a}$ from Equation (1) in Equation (2), we obtain $k=\left[\frac{2 n s^{2}}{v_{c} k_{1}}\right]\left[\frac{p i}{N t}\right]$ where

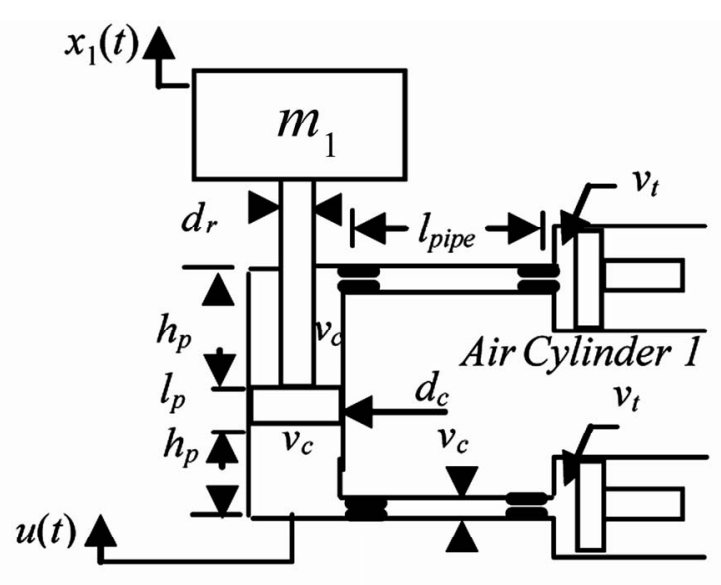

Air Cylinder 2

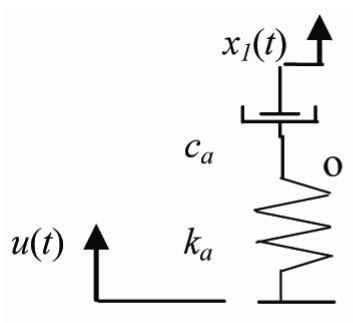

Maxwell Model

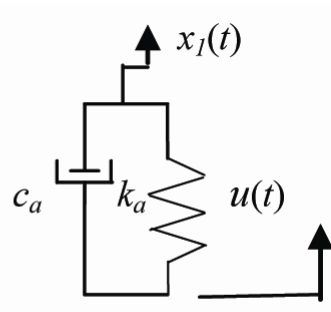

Vigot Model
Figure 1. (a) Cylinder-piston and air tank type system; (b) Mathematical models.

$$
s=\frac{\pi}{4}\left(d_{p}^{2}-d_{r}^{2}\right) \text { and } v_{c}=s h_{p}
$$

where $d_{p}$ is the piston diameter and $d_{r}$ is the piston rod diameter and $h_{p}$ is the height of the cylinder volume.

Defining the terms $p_{1}, p_{2}$ and $p_{3}$ as $p_{1}=\frac{d_{p}}{d_{c}}, p_{2}=\frac{d_{r}}{d_{c}}$ and $p_{3}=\frac{h_{p}}{d_{c}}$ and substituting the values of $p_{1}, p_{2}, p_{3}, s$ and $v_{c}$ in Equation (3), we obtain

$$
k=\frac{n \pi}{2 p_{3} k_{1}}\left[p_{1}^{2}-p_{2}^{2}\right]\left[d_{c}\right]\left[\frac{p i}{N t}\right]
$$

Assuming the values $p_{1}=0.985, p_{2}=0.333, p_{3}=0.5$ and $k_{1}=970 \mathrm{~N} / \mathrm{m}$, the Equation (4) becomes.

$$
k=0.00403146\left[d_{c}\right]\left[\frac{p i}{N t}\right]
$$

\subsubsection{Air Damping Ratio $\zeta_{a}[3]$}

$$
\zeta_{a}=\left[\frac{w_{a} v_{t}}{2 n c_{r} p i}\right] \text { where } w_{a}=\left[\frac{k_{a}}{m_{1}}\right]^{1 / 2}
$$

The capillary flow coefficient $c_{r}$ is given as

$$
c_{r}=\left[\frac{\pi\left(d_{\text {pipe }}\right)^{4}}{128 \mu_{o} l_{\text {pipe }}}\right] \text { (Refer [3]) }
$$

in which $d_{p i p e}$ and $l_{\text {pipe }}$ are the capillary pipe diameter and length respectively and $\mu_{o}$ is the viscosity of air at atmospheric temperature. Taking the value of $k_{a}$ from Equation (1) and substituting it in Equation (6), we get

$$
w_{a}=\left[\frac{2 n p i s^{2}}{v_{c} N t m_{1}}\right]^{1 / 2}=s \sqrt{\left[\frac{2 n}{v_{c} m_{1}}\right]} \sqrt{\left[\frac{p i}{N t}\right]}
$$

Substituting $w_{a}$ from Equation (8) and $c_{r}$ from Equation (7) in Equation (6)

we obtain

$$
\begin{aligned}
& \zeta_{a}=\left[\frac{\left[s \sqrt{\frac{2 n}{v_{c} m_{1}}} \sqrt{\frac{p i}{N t}}\right]\left[v_{c} N t\right]}{2 n\left[\frac{\pi\left(d_{\text {pipe }}\right)^{4}}{128 \mu_{o} l_{\text {pipe }}}\right] p i}\right] \text { or } \\
& \zeta_{a}=Q_{1}\left[\frac{l_{\text {pipe }}}{\left(d_{\text {pipe }}\right)^{4}}\right] \frac{1}{\sqrt{\frac{p i}{N t}}} \\
& Q_{1}=\left[\frac{128 \mu_{o} s}{\pi}\right]\left[\sqrt{\frac{v_{c}}{2 n m_{1}}}\right]
\end{aligned}
$$

where

Similarly using expressions (4) and (9) respectively 
for $k$ and $\zeta_{a}$ one can write

$$
\begin{gathered}
\zeta_{a}=Q_{2} \frac{1}{\sqrt{k}} \\
Q_{2}=\frac{w_{1}\left[\frac{1}{4}\left(d_{p}^{2}-d_{r}^{2}\right)\right]^{2}\left(128 \mu l_{\text {pipe }}\right)}{\left(d_{\text {pipe }}\right)^{4}}
\end{gathered}
$$

\subsection{Air Damper Characteristics}

From Equation (10), it is seen that $\zeta_{a}$ will be large for small values of $k$, i.e. for small values of $k_{a}$ for given value of $k$. To provide variable damping ratio, the value of $k$ can be varied. For the application of this device as a variable damping unit smaller values of $k(0.05$ to 0.125$)$ are preferred. Also $k$ depends on the ratio (pi/Nt), (refer Equation (4)) i.e. for small values of $k$, ratio (pi/Nt) should be kept small.

\subsubsection{Effect of the Cylinder Diameter $\boldsymbol{d}_{c}$ on Air Spring Rate Ratio $\boldsymbol{k}$ (Refer Figure 2)}

Here $d_{c}$ is varied as $d_{c}=10 \mathrm{~mm}, d_{c}=20.0 \mathrm{~mm}$ and $d_{c}=$ $30.0 \mathrm{~mm}$. The values of $d_{r}=(0.333)\left(d_{c}\right)=(0.333)(30.0)$ $=10 \mathrm{~mm}$ and $h_{p}=(0.5)\left(d_{c}\right)=(0.5)(30.0)=15 \mathrm{~mm}$ have been obtained. Considering a sliding fit between the piston and cylinder the piston diameter $d_{p}$ is taken as 29.95 $\mathrm{mm}$ for a cylinder diameter $d_{c}=30 \mathrm{~mm}$. Figure 2 shows the effect of variation of cylinder bore $d_{c}$ on spring rate ratio $k$.

\subsubsection{Effect of $\boldsymbol{d}_{\text {pipe }}$ on $\boldsymbol{\zeta}_{\boldsymbol{a}}$ (Refer Figure 3)}

Using the Equation (10), the effect of variation of ratio on air damping ratio $\zeta_{a}$ has been obtained for the values of $d_{\text {pipe }}=2.5,2.0$ and $1.5 \mathrm{~mm}$. with $l_{\text {pipe }}=3.0 \mathrm{~m}$. Figure 4 shows the effect of air spring rate ratio $k$ on the air damping ratio $\zeta_{a}$. for the values of $d_{\text {pipe }}=2.5,2.0$ and 1.5 $\mathrm{mm}$. with $l_{\text {pipe }}=3.0 \mathrm{~m}$.

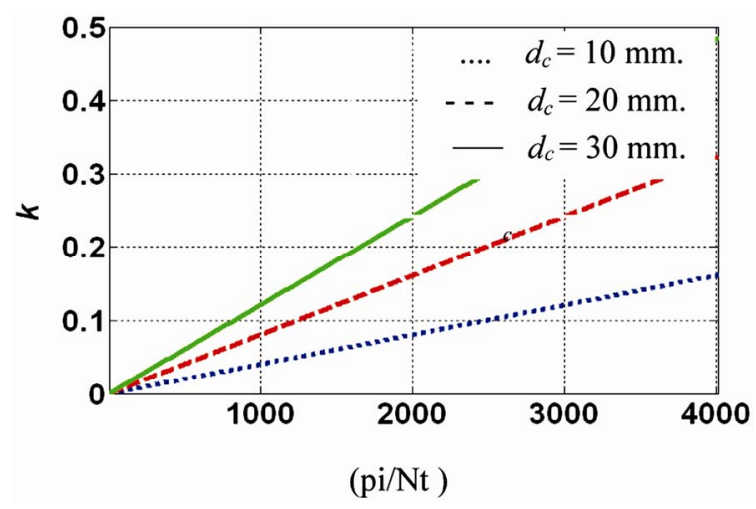

Figure 2. $k$ vs (pi/Nt) for different values of $d_{c}=$ a) $10 \mathrm{~mm}$, b) $20 \mathrm{~mm}$, c) $30 \mathrm{~mm}$.

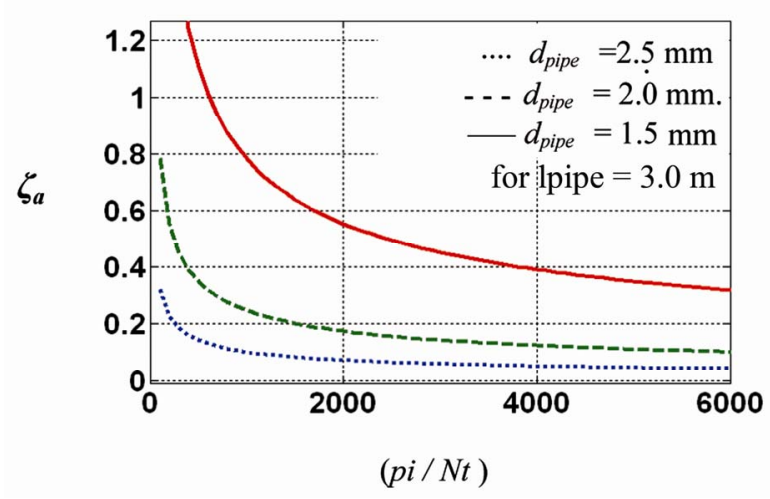

Figure 3. $\zeta_{a} v s(p i / N t)$ with $l_{p i p e}=3.0 \mathrm{~m} d_{p i p e}=$ a) $2.5 \mathrm{~mm}$, b) $2.0 \mathrm{~mm}, \mathrm{c)} 1.5 \mathrm{~mm}$.

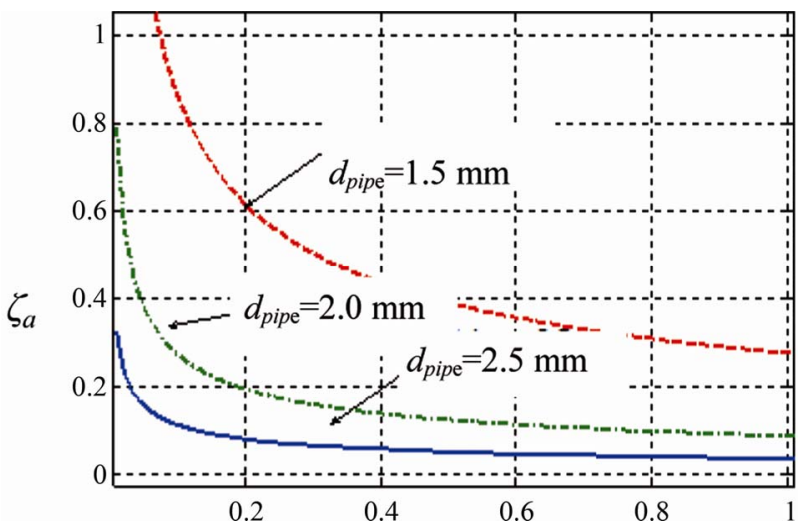

Figure 4. $\zeta_{a} v s k$ with $l_{\text {pipe }}=3.0 \mathrm{~m}$ and $d_{p i p}=$ a) $2.5 \mathrm{~mm}$, b) 2.0 $\mathrm{mm}, \mathrm{c)} 1.5 \mathrm{~mm}$.

From the curves of Figures 2, 3 and 4, it is seen that the developed air damper can provide appreciable increase in the damping ratio for values of the ratio $(p i / N t)$ in the range 500 to $6000 \mathrm{~N} / \mathrm{sq}$.m. per unit volume ratio $\left(v_{t} / v_{c}\right)$.

\subsubsection{Developed Air Damper Specifications}

Plate 1 shows the details of the air damper cylinder and slider assembly and air damper piston rod fitted to the sprung mass assembly. The air damper has been developed with the physical dimensions given in Table 1.

A double acting air cylinder configuration has been selected with the piston travel of $\pm 15 \mathrm{~mm}$ amplitude. The base excitation of $\pm 1.5 \mathrm{~mm}$ amplitude is provided. The material used for the entire assembly is steel with EN8 series, properly ground and finished to the selected dimensions. The sprung mass is in the form of a circular plate made up of C.I.

\subsubsection{SDOF Quarter Car Vehicle Suspension System Model [5]}

Thus the developed cylinder-piston and air tank-type air damper is capable of providing variable damping ratio. 


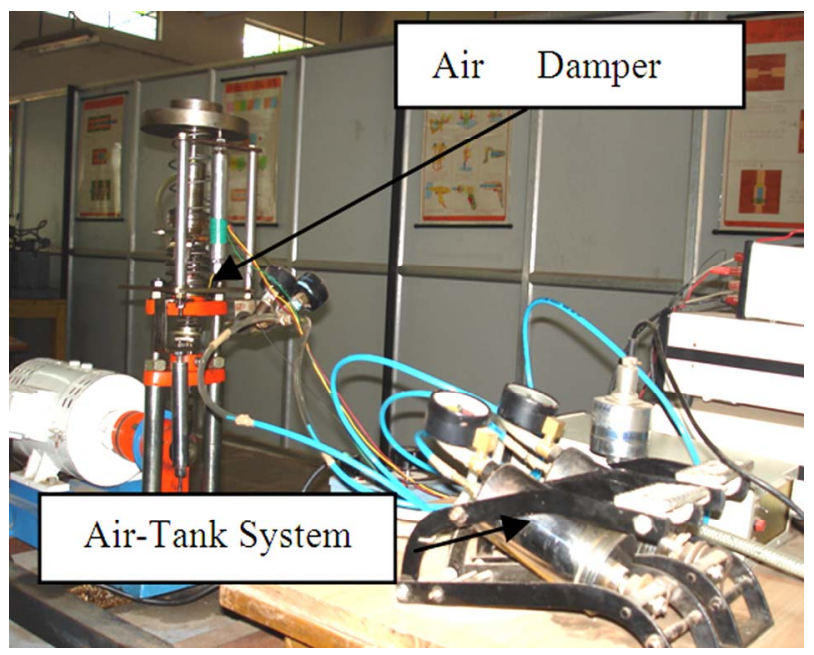

Plate 1. Air damper cylinder-piston and air-tank system.

Table 1. Air damper dimensions.

\begin{tabular}{lcccc}
\hline$d_{c}$ & $d_{p}$ & $d_{r}$ & $h_{p}$ & $l_{p}$ \\
\hline 30 & 29.85 & 10 & 15 & 13 \\
\hline
\end{tabular}

Figures 5(a) and (b) show a SDOF quarter-car vehicle suspension system model with system damping only and using the developed air damper respectively described in Section 2, respectively.

\subsection{Equations of Motion}

The equations of motion are given below

1) For Case 1 the equation of motion is

$$
m_{1} \ddot{x}_{1}=-k_{1}\left(x_{1}-u\right)-c_{1}\left(\dot{x}_{1}-\dot{u}\right)
$$

2) For Case 2 the equations of motion are

$$
\begin{gathered}
m_{1} \ddot{x}_{1}=-k_{1}\left(x_{1}-u\right)-c_{1}\left(\dot{x}_{1}-\dot{u}\right)-c_{a}\left(\dot{x}_{1}-\dot{y}\right) \\
-c_{a}\left(\dot{y}-\dot{x}_{1}\right)-k_{a}(\dot{y}-\dot{u})=0
\end{gathered}
$$

\subsection{Motion Transmissibility of the Sprung Mass}

Assume the steady state solutions of the Equations (11), (12) and (13) in the form $x_{1}=\mathrm{X}_{1} \mathrm{e}^{\mathrm{jwt}}, x_{2}=\mathrm{X}_{2} \mathrm{e}^{\mathrm{jwt}}$ and the base excitation as $u=\mathrm{Ue}^{\mathrm{jwt}}$, and following the usual procedure of solution, the expression for the motion transmissibility Mt1 (for the sprung mass) has been obtained for Case 1 as

$$
\mathrm{Mt} 1=\frac{X_{1}}{U}=\sqrt{\left[\frac{\left[A_{0}\right]^{2}+\left[A_{1} \lambda\right]^{2}}{\left[B_{0}-B_{2} \lambda^{2}\right]^{2}+\left[B_{1} \lambda\right]^{2}}\right]}
$$

where $A_{1}=2 \zeta_{1}, A_{0}=1, B_{2}=1, \mathrm{~B}_{1}=2 \zeta_{1}$ and $B_{0}=1$ and for Case 2 as

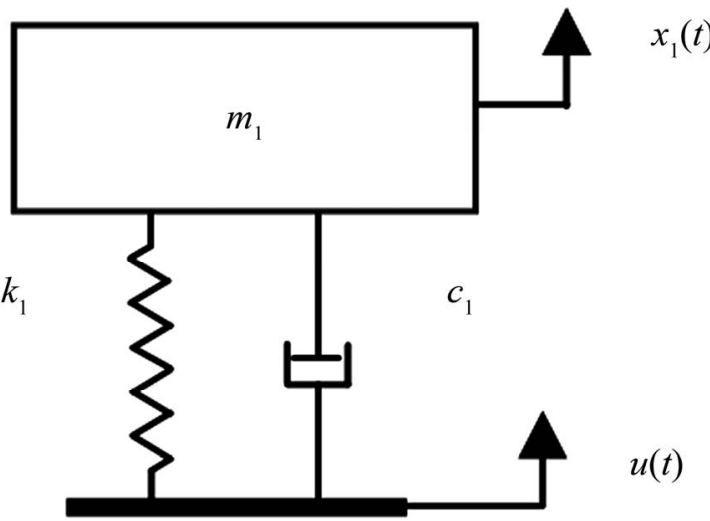

(a)

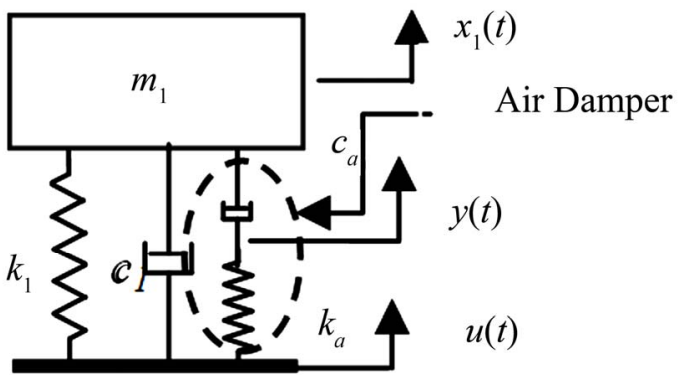

(b)

Figure 5. SDOF quarter-car vehicle suspension system model. (a) SDOF quarter-car vehicle suspension System with system damping; (b) SDOF quarter-car vehicle suspension system with system damping and air damper with maxwell type model.

$$
\mathrm{Mt} 1=\frac{X_{1}}{U}=\sqrt{\left[\frac{\left[-a_{2} \lambda^{2}+a_{0}\right]^{2}+\left[a_{1} \lambda\right]^{2}}{\left[b_{0}-b_{2} \lambda^{2}\right]^{2}+\left[-b_{3} \lambda^{3}+b_{1} \lambda\right]^{2}}\right]}
$$

where $a_{2}=2\left(\zeta_{1}+\sqrt{k} \zeta_{a}\right), a_{1}=\left(2 \zeta_{1} \delta+1+k\right), a_{0}=\delta, b_{3}$ $=1, b_{2}=\left(\delta+2 \zeta_{1}\right), b_{1}=\left(2 \zeta_{1} \delta+1+k\right)$ and $b_{0}=\delta$ where $\delta$ $=\left(\sqrt{k} / \zeta_{a}\right)$

Figure 6 and Figure 7 respectively show the curves of Mt1 vs $\lambda$ (where $\lambda$ is the ratio of excitation frequency $w$ to the undamped natural frequency $w_{1}$ of the system $\left(m_{1}, k_{1}\right)$ for Case 1 and Case 2 .

\subsection{Effect of Variation of Air Damper Spring Rate Ratio $k$}

The peak values of Mt1 (at resonance) for increasing values of the air spring rate ratio $k$ and the air damping ratio $\zeta_{a}$ are given in Table 2 (also refer Figure 6 and Figure 7). It is seen that as the value of air damper spring rate ratio $k$ and air damping ratio $\zeta_{a}$ increase, there is an appreciable reduction in the value of Mtl at the resonant frequency for the case where the air damper is modeled as a Maxwell type. 


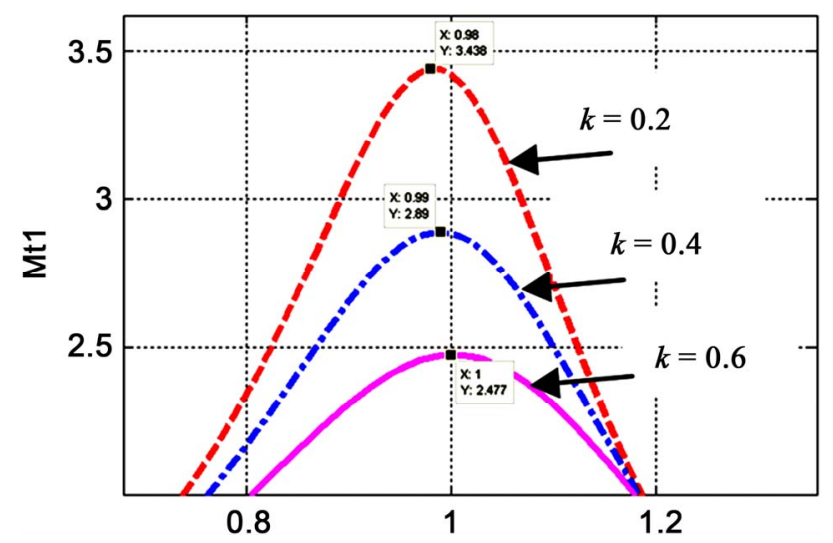

Figure 6. Mt1 vs $\lambda$ for $k=0.2,0.4$ and 0.6.

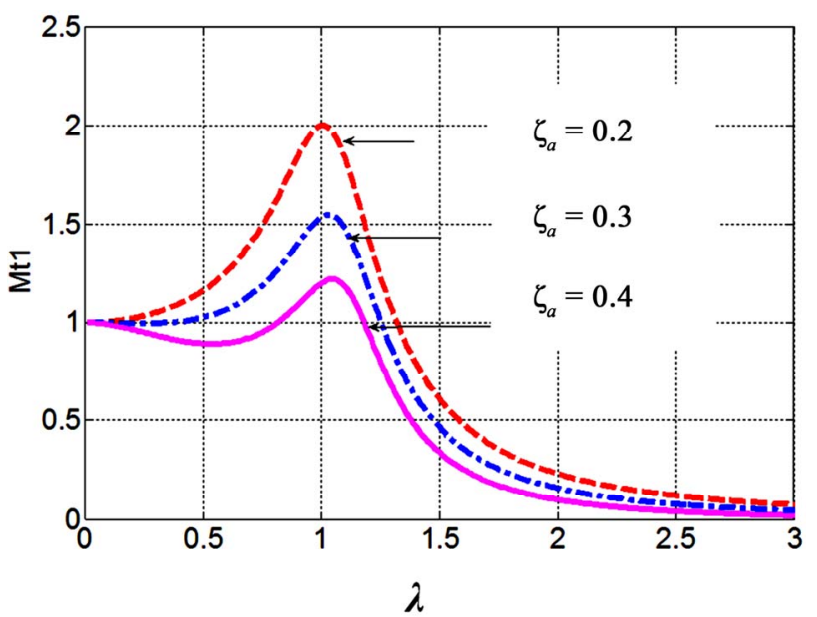

Figure 7. Mt1 vs $\lambda$ for $\zeta a=0.2,0.3$ and 0.4 .

Table 2. Values of spring rate ratio $k$ and damping ratio $\zeta_{a}$ varied with air damper modeled as a Maxwell Model.

\begin{tabular}{ccccccc}
\hline & \multicolumn{3}{c}{$\zeta_{1}=0.133, \zeta_{a}=0.3$} & \multicolumn{3}{c}{$\zeta_{1}=0.133, k=0.3$} \\
\cline { 2 - 7 } Peak Values of Mt1 & \multicolumn{3}{c}{$k$} & & \multicolumn{3}{c}{$\zeta_{a}$} \\
\cline { 2 - 7 } & 0.2 & 0.4 & 0.6 & 0.2 & 0.3 & 0.4 \\
\hline Mt1 & 3.48 & 2.89 & 2.48 & 2.0 & 1.55 & 2.92 \\
$\lambda$ & 0.92 & 0.96 & 0.98 & 0.98 & 1.08 & 1.15 \\
Figure No. & & 6 & & & 7 & \\
\hline
\end{tabular}

\section{Optimal Value $\zeta_{a o p t}$ of Air Damping Ratio $\zeta_{a}$}

The air damping is highly effective when the air damper is modeled as Maxwell type (Case 2 of Section 2). As such, Case 2 is taken for optimization of air damping ratio $\zeta_{a}$ The value of Mt1 given by Equation (15) is affected by system damping ratio $\zeta_{1}$ and the air damper characteristics i) air spring rate ratio $k$ and ii) air damping ratio $\zeta_{a}$.

Rearranging the equation as a function of $\zeta_{a}$, we obtain

$$
\mathrm{Mt} 1=\frac{X_{1}}{U}=\frac{A 2 \zeta_{a}{ }^{2}+A 1 \zeta_{a}+A 0}{B 2 \zeta_{a}{ }^{2}+B 1 \zeta_{a}+B 0}
$$

where $A 2, A 1, A 0, B 2, B 1, B 0$ are the constants containing frequency ratio $\lambda$, air spring rate ratio $k$ and system damping ratio $\zeta_{l}$. Differentiating the rearranged Equation (16) for Mt1 w.r.t. $\zeta_{a}$ and setting it equal to zero i.e. $\partial(\mathrm{Mt1}) / \partial\left(\zeta_{a}\right)=0$, we obtain a polynomial in terms of descending powers of $\zeta_{a}$ as

$$
\mathrm{C} 3 \zeta_{a}^{3}+C 2 \zeta_{a}^{2}+C 1 \zeta_{a}+C 0=0
$$

where $\mathrm{C}_{i}$ s are the constant coefficients containing $\zeta_{1}, k$ and $\lambda\left({ }_{i}=0,1,2\right.$ and 3$)$. The expressions derived for $\mathrm{C}_{\mathrm{i}} \mathrm{s}$ are lengthy and have not been included in the body of the write-up. The optimal value $\zeta_{\text {aopt }}$ of $\zeta_{a}$ is obtained by solving the Equation (17) and with the optimal value thus obtained, the values of Mt1 have been determined.

\section{Effect of Air Damping Ratio $\zeta_{a}$ on Amplitude Ratio} Mt1 for Various Values of Air Spring Rate Ratio $k$

The values of $\zeta_{\text {aopt }}$ for the air damper with a Maxwell type model have been obtained for $\zeta_{1}=0.133$ and $\lambda=1$ for different values of $k$. The minimum values of Mt1 (at resonance) for increasing values of the air spring rate ratio $k$ respectively are given in Table 3 (Also refer Figures 8 to 11).

\section{Experimental Setup}

Figure 13 shows the experimental setup designed and developed for dynamic response analysis of the SDOF quarter car suspension system model (refer also plate 2). The set up consists of a cam operated mechanism to provide sinusoidal base excitation of the desired amplitude and excitation frequency. The time dependant motion of both the base excitation $u(\mathrm{t})$ and the sprung mass response $x_{I}(t)$ are sensed and processed by the sensors consisting of LVDTs interfaced with a computer system. The software has been developed to process the input base excitation motion $u(\mathrm{t})$ vs time and the sprung mass response motion $x_{I}(\mathrm{t})$ vs time. The system also incorporates the facility to control the operating air pressure in the damper system through a computer interfaced system as shown in Figure 14. (Also refer Plate 2). Plate 2 shows all the details regarding the laboratory experimental model of a SDOF air damped SDOF quarter-car suspension system. The values of the sprung mass and suspension spring rate are taken respectively as $4.0 \mathrm{~kg}$ and $970 \mathrm{~N} / \mathrm{m}$.

\section{Air Pressure Control}

A computer interfacing system containing the closed loop air pressure control system associated with a set of two LVDTs to sense the suspension mass displacement $x_{1}(\mathrm{t})$ 
Table 3. Values of the air spring rate ratio $k$ varied with $\zeta_{1}$ $=0.133$ and $\lambda=1$.

\begin{tabular}{ccccccc}
\hline & \multicolumn{6}{c}{$k$} \\
\cline { 2 - 7 } & 0.025 & 0.050 & 0.075 & 0.100 & 0.200 & 0.300 \\
\hline $\mathrm{Mt1}_{(\min )}$ & 3.763 & 3.642 & 3.529 & 3.422 & 3.054 & 2.765 \\
$\zeta_{\text {aopt }}$ & 0.06 & 0.09 & 0.11 & 0.13 & 0.21 & 0.27 \\
Figure No. & & 8 & & & 9 & \\
& & & & & & \\
& & & & & & \\
$\mathrm{Mt1}_{\text {(min) }}$ & 2.538 & 2.356 & 2.209 & 2.089 & 1.989 & 1.905 \\
$\zeta_{\text {aopt }}$ & 0.33 & 0.38 & 0.44 & 0.48 & 0.52 & 0.57
\end{tabular}

Figure No.

$$
10
$$

11

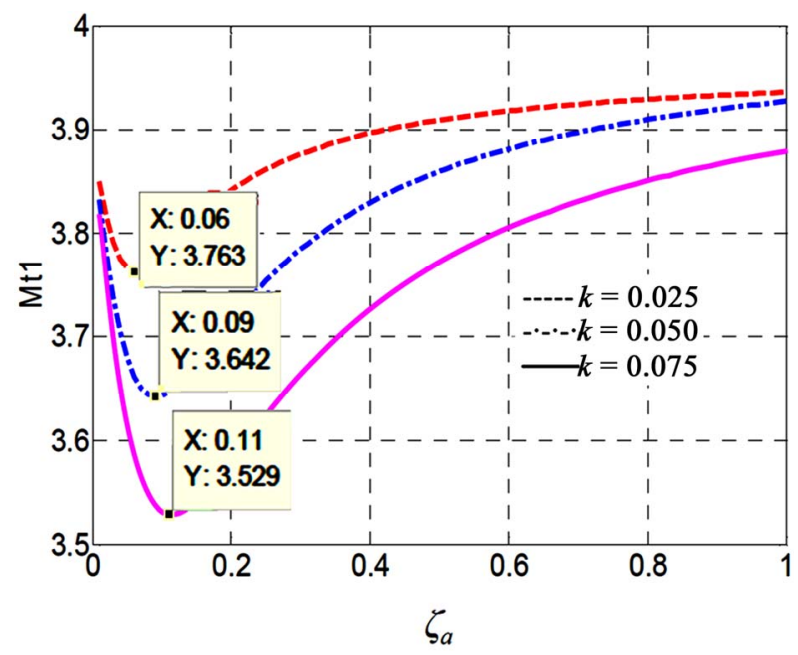

Figure 8. Mt1 vs $\zeta_{a}$ for $\boldsymbol{k}=\mathbf{0 . 0 2 5}, 0.05$ and 0.075 .

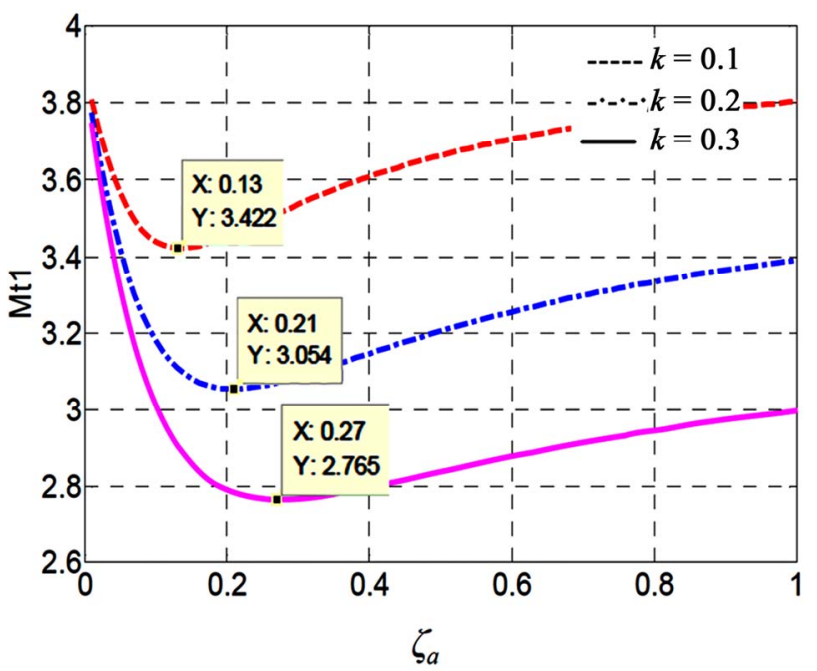

Figure 9. Mt1 vs $\zeta_{a}$ for $k=0.1,0.2$ and 0.3 .

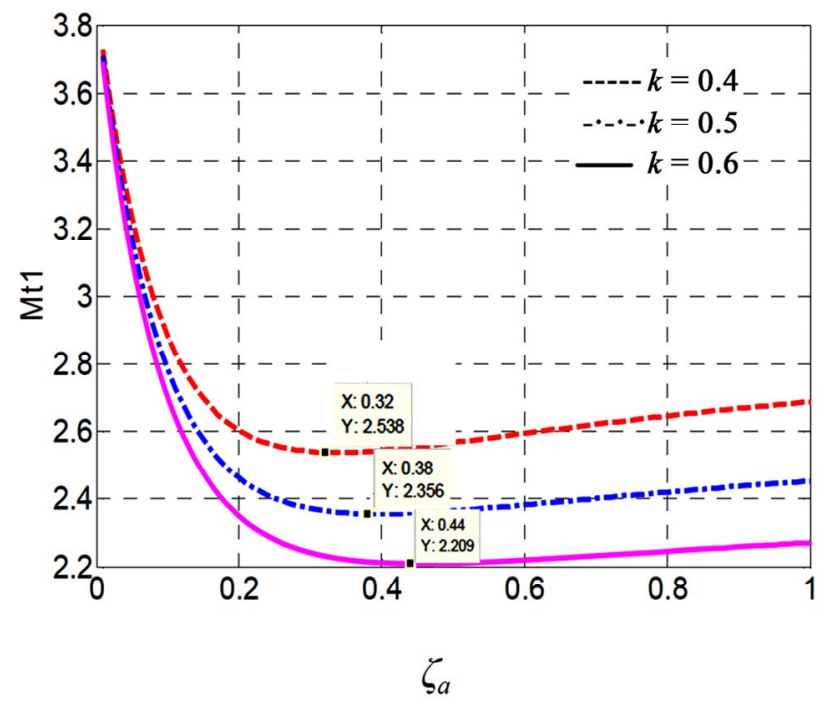

Figure 10. Mt1 vs $\zeta_{a}$ for $k=0.4,0.5$ and 0.6 .

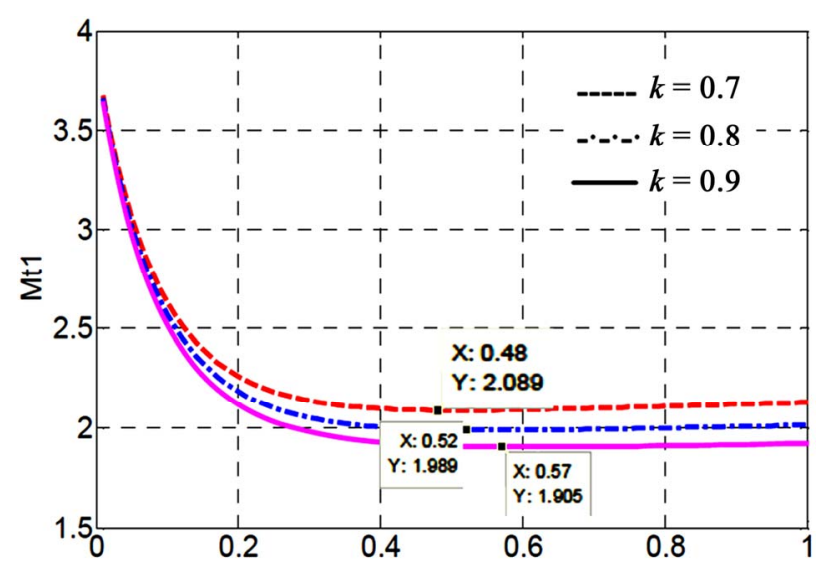

$\zeta_{a}$

Figure 11. Mt1 vs $\zeta_{a}$ for $k=0.7,0.8$ and 0.9 .

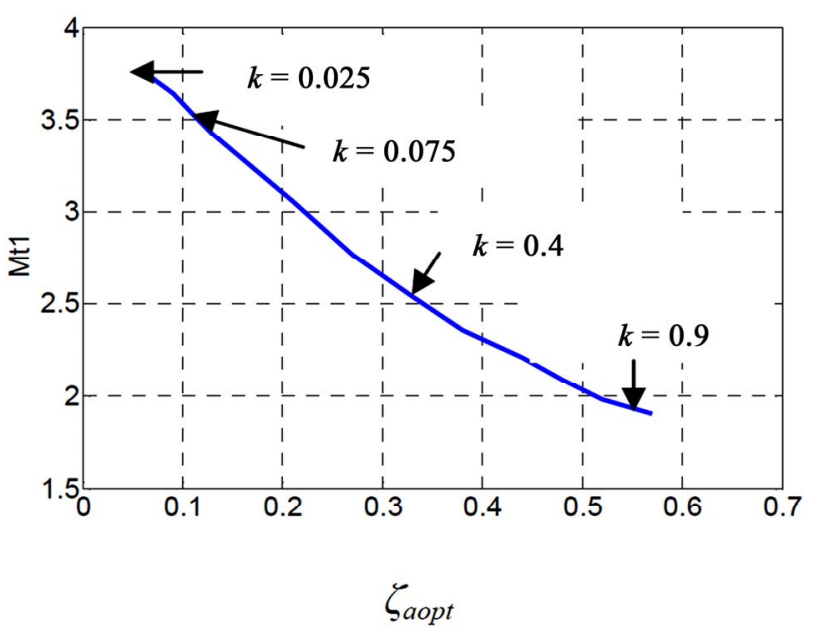

Figure 12. Mt1 vs $\zeta_{a}$ for $k=0.025$ to 0.9 . 


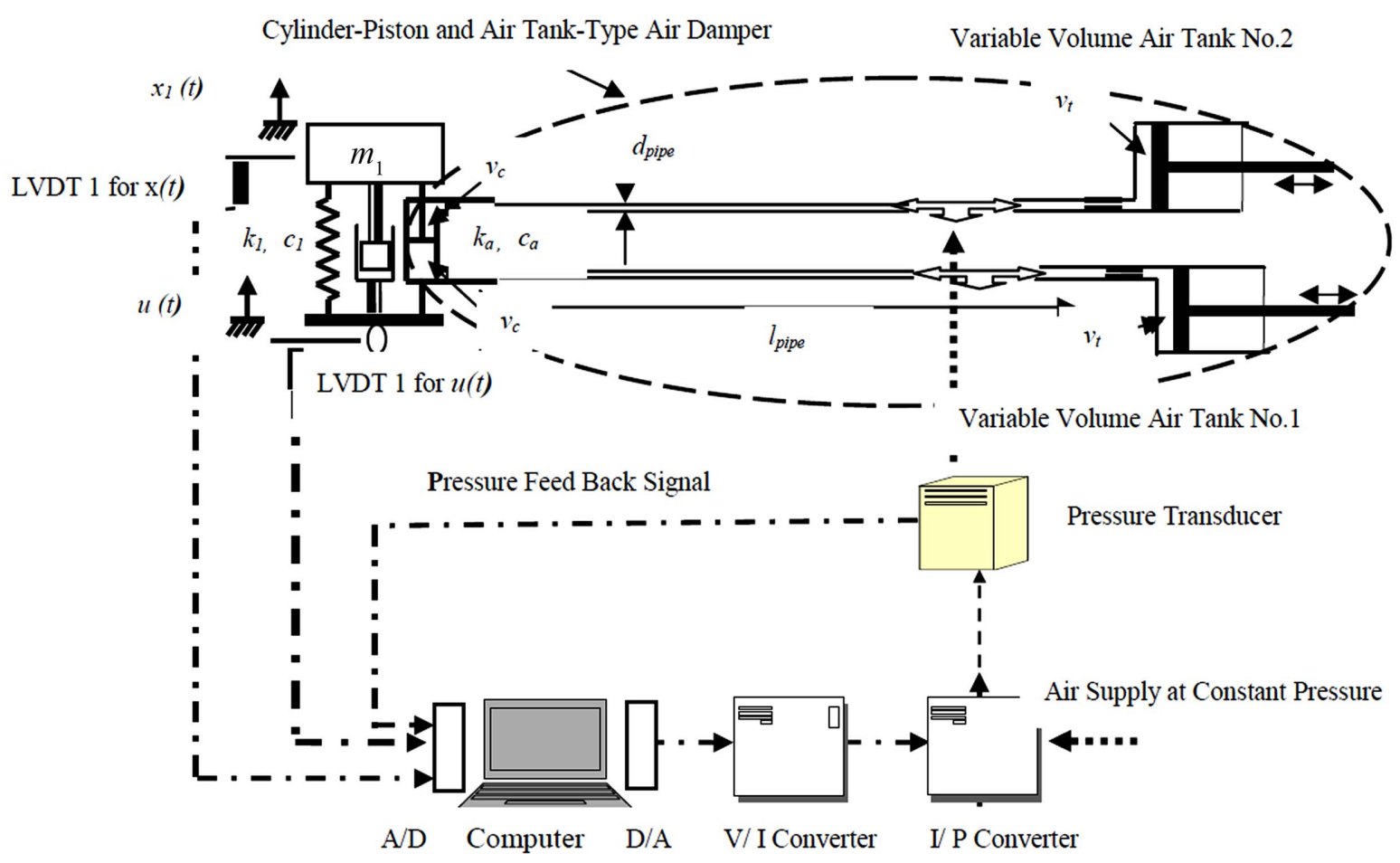

Figure 13. Experimental setup for an air damped SFOF suspension system model.

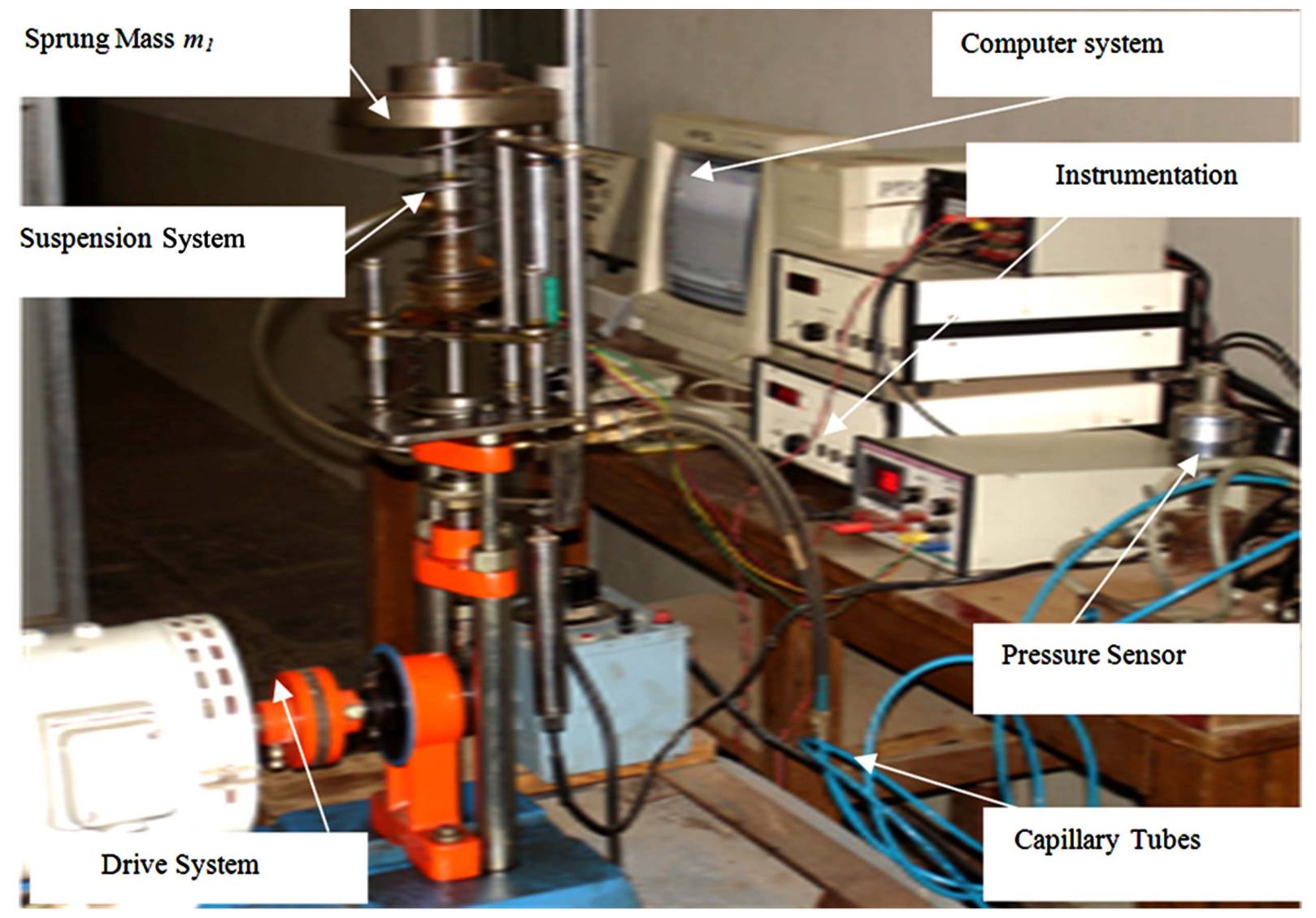

Plate 2. Experimental setup for a SFOF suspension system model with air damper. 


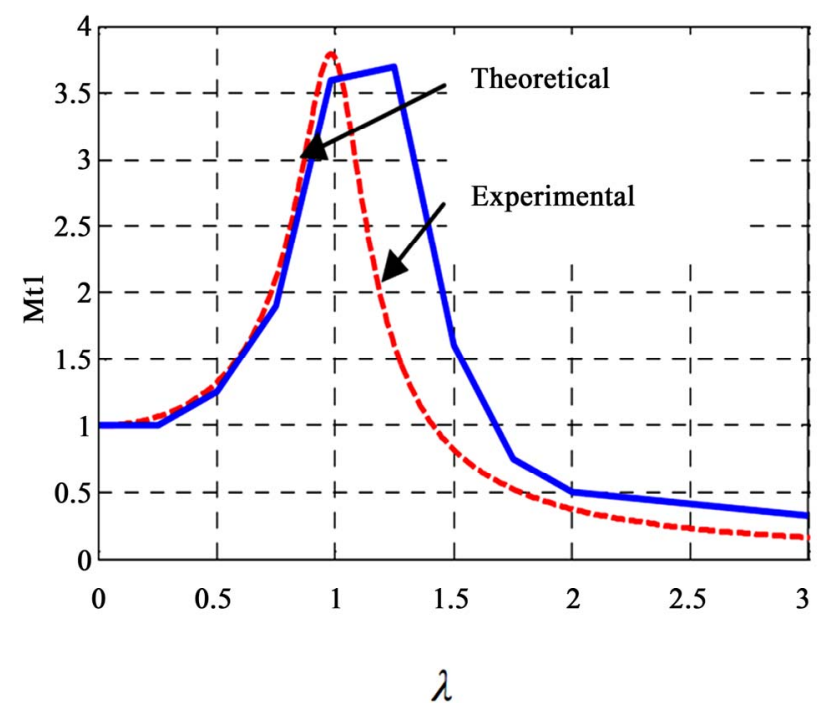

Figure 14. Mt1 vs $\lambda$ case (i) for $\zeta 1=0.133$.

and base excitation $u(\mathrm{t})$ has been developed .The ratio (pi/Nt) plays an important role in controlling the air damping ratio $\zeta_{a}$ in the system. The appropriate value of the ratio (pi/Nt), depending on the value of $\zeta_{a}$ desired in the system, can be set by controlling the value of operating air pressure $p i$ for a given value of ratio $N t=\left(\mathrm{v}_{\mathrm{t}} / \mathrm{v}_{\mathrm{c}}\right)$ or keeping the air pressure in the system at the atmospheric pressure and adjusting the value of the term $N t$ by adjusting the tank volume $v_{t}$.

\section{Experimental Analysis}

\subsection{Experimental Curves for Motion Transmissibility Mt1 vs Frequency Ratio $\lambda$}

Using the experimental setup shown in Figure13 and Plate 2 and by setting the appropriate values of the air spring rate ratio $k$ and the air damping ratio $\zeta_{a}$, the experimental plots of Mt1 vs $\lambda$ have been obtained for the SDOF system as i) With system damping only and without air damper (Refer Figure 14 and Table 4) ii) With system damping and air damper, with $k=0.2$ (Refer Figure 15 and Table 5).

\subsection{Experimental Motion Transmissibility Curves Mt1 vs $\lambda$, Using Optimal Values of Air Damping Ratio $\zeta_{\text {aopt }}$}

Table 6 shows the theoretical and experimental minimum values of motion transmissibility Mt1 at resonant frequency (with the air damper set for the optimal air damping ratio $\zeta_{\text {aopt }}$ at the value of $\zeta_{\text {aopt }}=0.33$ with air spring rate ratio $k=0.4$.
Table 4. Theoretical and Experimental Peak Values of Mt1 $\mathbf{1}_{(\max )}$ for the Case (i) for $\zeta_{1}=\mathbf{0 . 1 3 3}$.

\begin{tabular}{ccc}
\hline Peak value of & Theoretical & Experimental \\
\hline $\mathrm{Mt1}_{(\max )}$ & 3.798 & 3.70 \\
$\lambda$ & 0.98 & 1.25 \\
& \multicolumn{2}{c}{ Figure 14 } \\
\hline
\end{tabular}

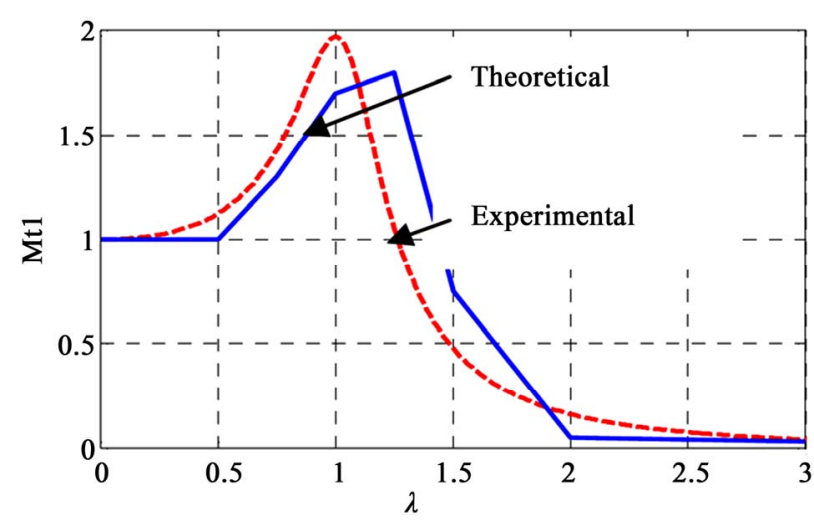

Figure 15. Mt1 vs $\lambda$ for $\zeta 1=0.133, k=0.2$ and $\zeta a=0.2$.

Table 5. Theoretical and experimental peak values of $\mathrm{Mt1}_{(\max )}$ for the case (ii) for $\zeta_{1}=0.133, k=0.2$ and $\zeta_{\mathrm{a}}=0.2$.

\begin{tabular}{ccc}
\hline Peak value of $\mathrm{Mt}_{(\max )}$ & Theoretical & Experimental \\
\hline $\mathrm{Mt}_{(\max )}$ & 1.89 & 1.80 \\
$\lambda$ & 0.98 & 1.29 \\
& Figure 15 & \\
\hline
\end{tabular}

Table 6. Theoretical and experimental peak values of $\mathrm{Mt}_{(\mathrm{mim})}$ for $\zeta_{1}=0.133, \lambda=1, k=0.4$ and $\zeta_{a o p t}=0.33$.

\begin{tabular}{ccc}
\hline $\mathrm{Mt}_{(\min )}$ & Theoretical & Experimental \\
\hline $\mathrm{Mt1}$ & 2.55 & 2.10 \\
$\lambda$ & 1.0 & 1.0 \\
& Figure 16 & \\
\hline
\end{tabular}

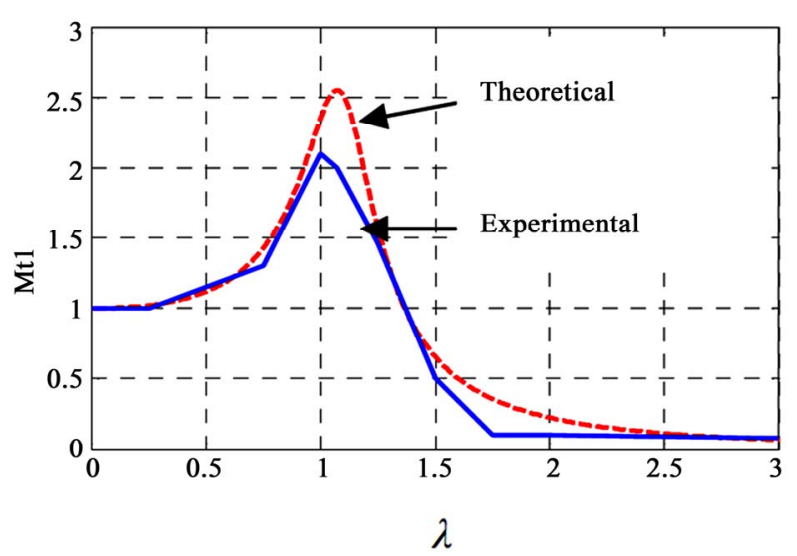

Figure 16. Mt1 vs $\lambda$ for $\zeta_{1}=0.133, \lambda=1, k=0.4$ and $\zeta_{\mathrm{a}}$ opt $=$ 0.33 . 


\section{Conclusions}

In this paper, a cylinder-piston and air-tank type air damper has been developed to provide variable air damping for a SDOF quarter car vehicle suspension system. The air damper has been based on the Maxwell type model. The effect of the air damper characteristics i.e. air damping ratio $\zeta_{a}$ and air spring rate ratio $k$ on the resonant response of an air damped SDOF vehicle suspension system has been analyzed. It is seen that as the value of the air spring rate ratio $k$ increases, the optimal value $\zeta_{a o p t}$ increases with decrease in the value of motion transmissibility Mt1. An experimental setup has been developed with an attendant air pressure control system. The values of $k$ and $\zeta_{a}$ for the air damper can be adjusted with the appropriate changes in dimensions of pipe length $l_{\text {pipe }}$, pipe diameter $d_{\text {pipe }}$ of capillary pipe between the air damper and the air tank and change in the ratio (pi/Nt). From the results of the experimental analysis shown in Figure 14 and Figure 15, it is seen that the experimental values of Mt1 are close to the corresponding theoretical values of Mt1. From Figure 16, it is seen that the theoretical and experimental minimum values of Mt1 for $\zeta_{\text {aopt }}=0.33$ with $k=0.4$ are in good agreement. The addition of the air damping improves substantially the motion transmis-

\section{Nomenclature}

$k_{1} \quad$ stiffness of spring supporting sprung mass

$m_{1} \quad$ sprung mass

$w_{1} \quad\left(\mathrm{k}_{1} / \mathrm{m}_{1}\right)^{1 / 2}$

$\zeta_{1} \quad$ system damping ratio

$w \quad$ applied frequency

$\lambda \quad$ frequency ratio $=\left(w / w_{1}\right)$

$d_{p} \quad$ piston diameter

$d_{c} \quad$ cylinder bore

$l_{p} \quad$ length of the piston

$h_{p} \quad$ height of bottom of piston from bottom of the

$h_{p} \quad$ cylinder

$d_{\text {pipe }} \quad$ inside diameter of the capillary pipe

$l_{\text {pipe }} \quad$ length of the capillary pipe

$\mu_{o} \quad$ viscosity of air sibility characteristics of the sprung mass of the SDOF quarter-car suspension model in the region of resonance.

\section{References}

[1] R.A. Williams, "Electronically Controlled Automotive Suspension Systems," Computing and Control Engineering Journal, Vol. 5, No. 3, 1994, pp. 143-148. doi: $10.1049 /$ cce: 19940310

[2] Toshihiko Asami and Nishihara, "Analytical and Experimental Evaluation of an Air Damped Dynamic Vibration Absorber: Design Optimizations of the Three-Element Type Model", Transaction of the ASME, Vol. 121, 1999, pp. 334-342.

[3] R. D. Cavanaugh, "Air Suspension Systems and ServoControlled Isolation Systems," Hand Book of Shock and Vibration, 2nd Edition, McGraw-Hill, New York, 1961, pp. 1-26

[4] R. G. Todkar and S. G. Joshi, "Some Studies on Transmissibility Characteristics of a 2DOF Pneumatic SemiActive Suspension System," Proceedings of International Conference on Recent Trends in Mechanical Engineering, Ujjain, 4-6 October 2007, pp. 19-28.

[5] P. Srinivasan, "Mechanical Vibration Analysis," Tata McHill Publishing Co., New Delhi, 1990. $n \quad$ index of expansion of the air

$k_{a} \quad$ stiffness of air spring

$k \quad$ spring rate ratio $=\left(\mathrm{k}_{\mathrm{a}} / \mathrm{k}_{1}\right)$

$w_{a} \quad\left(\mathrm{k}_{\mathrm{a}} / \mathrm{m}_{1}\right)^{1 / 2}$

coefficient of viscous damping provided by the

$c_{a} \quad$ air damper

$\zeta_{a} \quad$ air damping ratio of air spring

$\zeta_{\text {aopt }}$ optimal value of air damping ratio.

$u(t)$ base excitation

$x_{1}(t)$ dynamic displacement response of sprung mass

Mt1 motion transmissibility of the sprung mass $m_{1}$ 